X. Wang and G. Zhang

Nagoya Math. J.

Vol. 191 (2008), 135-148

\title{
MOST OF THE MAPS NEAR THE EXPONENTIAL ARE HYPERBOLIC
}

\author{
XIUMEI WANG AND GAOFEI ZHANG
}

\begin{abstract}
Let $f_{\lambda}(z)=\lambda e^{z}$. In this short note, we consider those maps $f_{\lambda}$ with $\lambda$ close to 1 . We show that the probability that $f_{\lambda}$ is hyperbolic approaches 1 as $\lambda \rightarrow 1$.
\end{abstract}

\section{$\S 1$. Introduction}

In 1980, Misiurewicz solved a 60-year-old conjecture due to Fatou by showing that the Julia set of the exponential map $z \mapsto e^{z}$ is the whole plane [10]. The dynamics of this map was then extensively studied by many authors. For instance, R. Devaney showed that the exponential map is not stable [3]. M. Rees showed that it is not recurrent [13], whereas E. Ghys, L. Goldberg, and D. Sullivan showed that it is so under a larger equivalence relation [8]. Almost at the same time, M. Lyubich showed that it is not ergodic and has wandering sets with positive Lebesgue measure [9].

Let $f_{\lambda}(z)=\lambda e^{z}$. The aim of this paper is to investigate what happens when one slightly perturbs the map $z \mapsto e^{z}$ in the family $f_{\lambda}, \lambda \in \mathbb{C} \backslash\{0\}$. It was proved by Devaney in [3] that in any small neighborhood of 1 , there is a $\lambda$ such that $f_{\lambda}$ is hyperbolic. Here we say $f_{\lambda}$ is hyperbolic if $f_{\lambda}$ has an attracting periodic cycle (Such a periodic cycle once exists, must be unique and necessarily attracts the orbit of the origin, see [15]). In this paper, we will show that the density of such hyperbolic parameters $\lambda$ approaches 1 as $\lambda \rightarrow 1$. Before the formulation of the Main Theorem, let us introduce some notations first.

For $r>0$ and $x \in \mathbb{C}$, let

$$
S_{r}(x)=\{z \in \mathbb{C} \mid \max \{|\Re(z-x)|,|\Im(z-x)|\}<r\}
$$

Received May 9, 2007.

Revised December 20, 2007.

2000 Mathematics Subject Classification: 58F23, 37F10, 37F45, 32H50, 30D05.

The second author is partially supported by NJU-0203005116 and National Basic Research Program of China (973 Program) (2007CB814800). 
denote the square with center $x$ and side length $2 r$. For a bounded set $X \subset \mathbb{C}$, let area $(X)$ and $\operatorname{diam}(X)$ denote the Euclidean area and Euclidean diameter of $X$, respectively. For $\lambda \in \mathbb{C} \backslash\{0\}$, let $J_{\lambda}$ denote the Julia set of $f_{\lambda}$. Let

$$
H=\left\{\lambda \in \mathbb{C} \backslash\{0\} \mid f_{\lambda} \text { is hyperbolic }\right\} .
$$

The main result of the paper is

Main Theorem. $\operatorname{area}\left(S_{r}(1) \cap H\right) / \operatorname{area}\left(S_{r}(1)\right) \rightarrow 1$ as $r \rightarrow 0$.

By the Main Theorem, it follows that if one takes randomly a parameter value $\lambda$, the probability that $J_{\lambda}=\mathbb{C}$ is small provided that $\lambda$ is near 1 . This leads us to pose the following question:

Question. Is there a positive measurable set $\Lambda \subset \mathbb{C} \backslash\{0\}$ such that $J_{\lambda}=\mathbb{C}$ for every $\lambda \in \Lambda$ ?

For rational maps, the question has a positive answer by M. Rees. The reader may refer to [14] for the construction of the set $\Lambda$. But the method used in [14] does not apply for the exponential family because of the presence of an essential singularity at the infinity. It is also interesting to note that a negative answer of the question would imply that hyperbolic maps are dense in the exponential family.

The following is the structure of the proof.

In Section 2, we show that there is a $C>0$ such that for any $\epsilon>0$, there is an $r_{0}>0$, such that for all $0<r<r_{0}$, there is a subset $\Lambda_{r}(1) \subset S_{r}(1)$ satisfying the following two conditions,

1. $\operatorname{area}\left(\Lambda_{r}(1)\right)>(1-C \epsilon) \operatorname{area}\left(S_{r}(1)\right)$, and

2. for every $\lambda \in \Lambda_{r}(1), f_{\lambda}$ is hyperbolic.

(Lemma 2.8). The strategy used in this step is the so called "parameter exclusion", which was first used by McMullen in [11], and later adapted by other authors in some particular situations (For examples, see [12] and [16]).

In Section 3, we prove that for every $r>0$ small, and every $\lambda \in \Lambda_{r}(1)$, the map $f_{\lambda}$ has an attracting periodic cycle (Lemma 3.1). The construction used in this step is implicitly contained in Devaney's proof in [3]. A similar construction may also be found in [1] and [5].

The Main Theorem then follows from Lemmas 2.8 and 3.1.

For more relative knowledge about the dynamics of the exponential maps, we refer the reader to [2], [6], [4], [7], and [17]. 


\section{§2. The set $\Lambda_{r}(1)$}

\subsection{Notations}

Let $\mathbb{N}$ denote the set of the positive integers. Let $a$ and $b$ be two positive quantities. We use $a \preceq b$ and $a \succeq b$ to mean that the ratio $a / b$ has a universal positive upper bound and a positive lower bound, respectively. We use $a \asymp b$ to mean that both such bounds exist.

\subsection{The Construction of $\Lambda_{r}(1)$}

For $n \in \mathbb{N}$, define $E_{n}(\lambda)=f_{\lambda}^{n}(0)$. Take $0<\delta<1 / 16$ and let it be fixed throughout the following. Let $r>0$ be small. Recall that $S_{r}(1)$ is the square with center 1 and side length $2 r$. Let $X_{0}=S_{r}(1)$ and for $n \geq 1$, define $X_{n}=E_{n}\left(S_{r}(1)\right)$. Then there is a least integer $n>0$ such that

$$
\operatorname{diam}\left(X_{n+1}\right)>\delta \pi
$$

By the choice of $n$, it follows that $\operatorname{diam}\left(X_{n}\right) \leq \delta \pi$. Since $\delta$ is fixed, $n$ depends only on $r$. To simplify the notation, let us simply write it as $n$ instead of $n(r)$.

Lemma 2.1. Let $M>1$ be large. Then for all $r>0$ small enough, there is an $R>\sqrt{2} r$ such that

$$
\bmod \left(B_{R}(1) \backslash S_{r}(1)\right)>M
$$

and then $E_{n}$ is univalent in $B_{R}(1)$ where $B_{R}(1)$ is the Euclidean disk with center 1 and radius $R$.

Proof. Let $K>0$ be an arbitrary number. Let $R>0$ be such that

$$
E_{n}(1+R)=E_{n}(1)+K \delta
$$

We claim

$$
\lim _{r \rightarrow 0} \sup _{\lambda \in B_{R}(1)}\left|\frac{D E_{n}(\lambda)}{D E_{n}(1)}-1\right|=0 .
$$

It follows that the map $E_{n}$ is like a linear map in $B_{R}(1)$ when $r>0$ is small. In particular, $E_{n}$ is univalent in $B_{R}(1)$ for all $r>0$ small enough. Since $\operatorname{diam}\left(X_{n}\right)<\delta$, it follows that

$$
R / r \succeq K
$$


and therefore $\bmod \left(B_{R}(1) \backslash S_{r}(1)\right)$ can be as large as possible provided $K$ is large enough. The lemma thus follows.

Now it is sufficient to prove the claim. By a direct calculation, we have

$$
D E_{n}(\lambda)=\frac{1}{\lambda} \sum_{k=1}^{n-1} P_{k}(\lambda)+P_{n-1}(\lambda)
$$

where

$$
P_{k}(\lambda)=\prod_{i=n-k+1}^{n} E_{i}(\lambda)
$$

Obviously we have

(a) $D E_{k}(\lambda)>D E_{k}(1)$ for any $\lambda>1$ and $k \in \mathbb{N}$,

(b) $D E_{k+1}(\lambda)>D E_{k}(\lambda)$ for any $\lambda>1$ and $k \in \mathbb{N}$,

(c) $D E_{k}(1)>2^{k-1}$ for $k \in \mathbb{N}$.

It is also clear that

$$
E_{n}(1)>2^{n-1}
$$

From (2), (a), and (c), we have

$$
R<K \delta / D E_{n}(1)<K \delta / 2^{n-1} .
$$

Note that $E_{n}(1+R)=(1+R) e^{E_{n-1}(1+R)}$, we have

(7) $1+\frac{E_{n}(1+R)-E_{n}(1)}{E_{n}(1)}=\frac{E_{n}(1+R)}{E_{n}(1)}=(1+R) e^{E_{n-1}(1+R)-E_{n-1}(1)}$.

Since $E_{n}(1+R)-E_{n}(1)=K \delta$ and $n \rightarrow \infty$ as $r \rightarrow 0$, from (5), (6), and (7), we have

$$
\left|E_{n-1}(1+R)-E_{n-1}(1)\right| \preceq K \delta / 2^{n} .
$$

From (b) and (8), we have

$$
\left|E_{k}(1+R)-E_{k}(1)\right| \preceq K \delta / 2^{n}
$$

for all $1 \leq k<n-1$. 
Now from (3) we need only to prove that as $r \rightarrow 0, P_{k}(\lambda) / P_{k}(1) \rightarrow 1$ uniformly for $1 \leq k \leq n-1$. In fact, for $1 \leq k \leq n-1$,

$$
\left|\frac{P_{k}(\lambda)}{P_{k}(1)}-1\right|=\left|\prod_{i=n-k+1}^{n}\left(1+\frac{E_{i}(\lambda)-E_{i}(1)}{E_{i}(1)}\right)-1\right| \preceq \sum_{i=1}^{n} \frac{\left|E_{i}(\lambda)-E_{i}(1)\right|}{E_{i}(1)} .
$$

By (3), $\left|D E_{k}(1+z)\right| \leq D E_{n}(1+|z|)$ holds for all for $|z|<R$ and $k \geq 1$, so we have for all $\lambda \in B_{R}(1)$,

$$
\left|E_{i}(1)-E_{i}(\lambda)\right| \leq E_{i}(1+R)-E_{i}(1) .
$$

This, together with (5), (8), (9), and the fact that $n \rightarrow \infty$ as $r \rightarrow 0$, implies that

$$
\left|\frac{P_{k}(\lambda)}{P_{k}(1)}-1\right| \preceq n K \delta / 2^{n} \rightarrow 0 \text { as } r \rightarrow 0 .
$$

This proves the claim and thus completes the proof of the lemma.

Let $\Phi_{X_{n}}: X_{n} \rightarrow S_{r}(1)$ be the inverse branch of $E_{n}$ which maps $X_{n}$ to $S_{r}(1)$. Recall that $0<\delta<1 / 16$ is the value taken in (1) and is fixed throughout the paper.

LEMma 2.2. Let $r>0$ be small. Then there is some $R>r$ such that $\bmod \left(S_{R}(1) \backslash S_{r}(1)\right)>m(\delta)$ where $m(\delta)>0$ depends only on $\delta$ and $E_{n+1}$ is univalent in $S_{R}(1)$.

Proof. Let us take $K=1 / \sqrt{\delta}$ in (2). By the claim immediately after (2), the assumption that $0<\delta<1 / 16$, and the fact that $\operatorname{diam}\left(X_{n}\right)<\delta$, the map $\Phi_{X_{n}}$ can be defined in the larger square $S_{\sqrt{\delta} / 2}\left(E_{n}(1)\right) \supset X_{n}$ for all $r>0$ small enough.

For $\xi \in S_{\sqrt{\delta} / 4}\left(E_{n}(1)\right)$, let $\lambda=\Phi_{X_{n}}(\xi)$. Since $E_{n+1}(\lambda)=\lambda e^{E_{n}(\lambda)}$ and $X_{n} \subset S_{\sqrt{\delta} / 4}\left(E_{n}(1)\right)$ (This is because $\sqrt{\delta} / 4>\delta$ ), the lemma follows if we can prove that for all $r>0$ small enough, the map $F_{n}(\xi)=E_{n+1}(\lambda)=$ $\Phi_{X_{n}}(\xi) e^{\xi}$ is univalent in $S_{\sqrt{\delta} / 4}\left(E_{n}(1)\right)$. To see this, first note that $F_{n}^{\prime}(\xi)=$ $e^{\xi}\left(\Phi_{X_{n}}(\xi)+\Phi_{X_{n}}^{\prime}(\xi)\right)$. Since $\Phi_{X_{n}}^{\prime}\left(E_{n}(1)\right)=1 / D E_{n}(1) \rightarrow 0$ as $r \rightarrow 0$, from Koebe's distortion theorem, it follows that as $r \rightarrow 0, \Phi_{X_{n}}^{\prime}(\xi) \rightarrow 0$ and $\Phi_{X_{n}}(\xi) \rightarrow 1$ uniformly for $\xi \in S_{\sqrt{\delta} / 4}\left(E_{n}(1)\right)$. This implies

$$
\lim _{r \rightarrow 0} \sup _{\xi, z \in S_{\sqrt{\delta} / 4}\left(E_{n}(1)\right)}\left|\frac{F_{n}^{\prime}(\xi)}{F_{n}^{\prime}(z)}-1\right| \leq \lim _{r \rightarrow 0} \sup _{\xi, z \in S_{\sqrt{\delta} / 4}\left(E_{n}(1)\right)}\left|e^{\xi-z}-1\right|<\sqrt{\delta}<1 / 4 .
$$

It thus follows that for all $r>0$ small, $F_{n}$ is univalent in $S_{\sqrt{\delta} / 4}\left(E_{n}(1)\right)$. This completes the proof of the lemma. 
Let $0<\epsilon<1 / 80$ be an arbitrary small number. Let $\Gamma_{\epsilon}$ denote the family of the horizontal straight lines $\Im(z)=k \epsilon$ and the vertical straight lines $\Re(z)=k \epsilon$ for $k \in \mathbb{Z}$. It follows that the lines in $\Gamma$ divide the complex plane into infinitely many squares with side length $\epsilon$. Let us call each of these squares an $\epsilon$-square.

Define

$$
A_{\epsilon}=\{z \in \mathbb{C} \mid(2 k+1 / 2) \pi+\epsilon<\Im(z)<(2 k+3 / 2) \pi-\epsilon \text { for some } k \in \mathbb{Z}\}
$$

and

$$
B_{\epsilon}=\{z \in \mathbb{C} \mid(2 k-1 / 2) \pi+\epsilon<\Im(z)<(2 k+1 / 2) \pi-\epsilon \text { for some } k \in \mathbb{Z}\} .
$$

Let $S$ be an $\epsilon$-square. Let $c$ be the center of $S$. Then $S=S_{\epsilon / 2}(c)$. We call $S$ an expanding square of generation 1 if $S_{\epsilon}(c) \subset X_{n+1} \cap B_{\epsilon}$ and a contracting square of generation 1 if $S_{\epsilon}(c) \subset X_{n+1} \cap A_{\epsilon}$ (Note that $S$ is well contained in $S_{\epsilon}$, that is, there is a definite space around $S$ in $S_{\epsilon}(c)$ ). From now on, we call $S_{\epsilon}(c)$ the $\epsilon$-neighborhood of $S$.

Note that by Lemma 2.2, for any expanding square $S=S_{\epsilon / 2}(c)$ of generation 1 , there is an inverse branch of $E_{n+1}$, say $\Phi_{S}$, which is defined in the larger square $S_{\epsilon}(c)$, such that $\Phi_{S}$ maps $S$ into $S_{r}(1)$. Let us call $\Phi_{S}$ the associated map to the expanding square $S$.

LEMMA 2.3. There is a $r_{0}>0$ such that for all $0<r<r_{0}$, the map $E_{n+2} \circ \Phi_{S}$ can be univalently defined in the $\epsilon$-neighborhood of $S$.

Proof. It is clear that the map $E_{n+2} \circ \Phi_{S}$ is holomorphic in $S_{\epsilon}(c)$. Let us show that it is univalent. To see this, note that for $\xi \in S_{\epsilon}(c)$,

$$
E_{n+2} \circ \Phi_{S}(\xi)=\Phi_{S}(\xi) e^{\xi} .
$$

We thus have

$$
D\left(E_{n+2} \circ \Phi_{S}\right)(\xi)=\left(\Phi_{S}^{\prime}(\xi)+\Phi_{S}(\xi)\right) e^{\xi} .
$$

From Lemma 2.2, it follows that there is an $r_{0}>0$ small, such that when $0<r<r_{0},\left|\Phi_{S}^{\prime}(\xi)\right|<1 / 100$ and $\left|\Phi_{S}(\xi)-1\right|<1 / 100$. We thus have

$$
\sup _{\xi, z \in S_{\epsilon}(c)}\left|\frac{\left(E_{n+2} \circ \Phi_{S}\right)^{\prime}(\xi)}{\left(E_{n+2} \circ \Phi_{S}\right)^{\prime}(z)}-1\right|<\frac{51}{49} \sup _{\xi, z \in S_{\epsilon}(c)}\left|e^{\xi-z}-1\right|<20 \epsilon<1 / 4 .
$$

The last two inequalities come from the assumption that $0<\epsilon<1 / 80$ and the fact that $\left|e^{z}-1\right|<4|z|$ for $|z|<1 / 4$. This implies that the map $E_{n+2} \circ \Phi_{S}$ is univalent in $S_{\epsilon}(c)$ and completes the proof of the lemma. 
The proof of the following lemma is direct and we shall leave it to the reader.

Lemma 2.4. Let $0<\epsilon<1 / 80$. Then there is a $K>100$ such that for any $\epsilon$-square $S \subset B_{\epsilon}$ with $\inf _{z \in S} \Re(z)>K$, the following inequality holds

$$
\Re\left(\lambda e^{z}\right)>2 \Re(z)
$$

for any $z \in S$ and $\lambda \in S_{1 / 32}(1)$.

Now we assume that $0<r<r_{0}$ for some $r_{0}>0$ small enough. We will define expanding and contracting squares by induction. Let $k \geq 1$. Suppose all the expanding squares $S=S_{\epsilon / 2}(c)$ of generation $k$ and their associated maps $\Phi_{S}$ are defined such that

1. $\Phi_{S}$ is defined in the $\epsilon$-neighborhood of $S$ and maps $S$ into $S_{r}(1)$,

2. $\inf _{z \in S} \Re(z)>K$ where $K$ is the constant in Lemma 2.4,

3. $\left|\Phi_{S}^{\prime}(z)\right|<1 / 100$ for every $z \in S$,

4. the map $E_{n+k+1} \circ \Phi_{S}$ is univalent in the $\epsilon$-neighborhood of $S$.

An $\epsilon$-square $T$ is called an expanding square generated by $S$ if its $\epsilon$ neighborhood is contained in $B_{\epsilon} \cap\left(E_{n+k+1} \circ \Phi_{S}\right)(S)$. Similarly, An $\epsilon$-square $T$ is called an contracting square generated by $S$ if its $\epsilon$-neighborhood is contained in $A_{\epsilon} \cap\left(E_{n+k+1} \circ \Phi_{S}\right)(S)$. All the expanding squares and contracting squares generated by expanding squares of generation $k$ are called of generation $k+1$. To each expanding square $T$ of generation $k+1$, we define the associated map $\Phi_{T}$ by

$$
\Phi_{T}=\Phi_{S} \circ\left(E_{n+k+1} \circ \Phi_{S}\right)^{-1} .
$$

LEMMA 2.5. We have

1. $\Phi_{T}$ is defined in the $\epsilon$-neighborhood of $T$ and maps $T$ into $S_{r}(1)$,

2. $\inf _{z \in T} \Re(z)>K$ where $K$ is the constant in Lemma 2.4,

3. $\left|\Phi_{T}^{\prime}(z)\right|<1 / 100$ for every $z \in T$,

4. the map $E_{n+k+2} \circ \Phi_{T}$ is univalent in the $\epsilon$-neighborhood of $T$.

Proof. The first assertion follows from equation (11). The second assertion follows from Lemma 2.4 by assuming that $0<r<1 / 32$. To verify 
the third assertion, note that for $z \in S,\left(E_{n+k+1} \circ \Phi_{S}\right)(z)=\Phi_{S}(z) e^{z}$. It follows that

$$
\left(E_{n+k+1} \circ \Phi_{S}\right)^{\prime}(z)=e^{z}\left(\Phi_{S}^{\prime}(z)+\Phi_{S}(z)\right) .
$$

By induction, $\Phi_{S}^{\prime}(z)+\Phi_{S}(z)$ is close to 1 and $\left|e^{z}\right|$ is large. It follows that for any $\xi \in T,\left|D\left(E_{n+k+1} \circ \Phi_{S}\right)^{-1}(\xi)\right|$ is small. The third assetion then follows from the induction and the chain rule. The proof of the last assertion uses the same argument as in the proof of Lemma 2.3 and the previous three assertions.

From Lemma 2.5, it follows that there is an $r_{0}>0$ such that for all $0<r<r_{0}$, the expanding squares and contracting squares can be defined inductively for generations of all $k \geq 1$.

Remark 2.1. By the construction of the expanding squares $S$ and their associated maps $\Phi_{S}$, it follows that $\Phi_{S}$ can be defined in the $\epsilon$-neighborhood of $S$, and thus has uniform distortion in $S$.

Definition 2.1. Let $\Lambda_{r}(1)$ be the set which consists of all the points $z$ in $S_{r}(1)$ for which there exist an integer $k \geq 1$ and expanding squares $S_{i}$, $1 \leq i \leq k-1$, and a contracting square $S_{k}$, such that $S_{i}$ is generated by $S_{i-1}$ for $2 \leq i \leq k$, and $E_{n+i}(z) \in S_{i}$ for all $1 \leq i \leq k$.

\subsection{The area of $\Lambda_{r}$}

LEMma 2.6. There is a uniform $C>0$ such that for all $r>0$ small enough,

$$
\operatorname{area}(R) / \operatorname{area}\left(X_{n+1}\right)<C \epsilon
$$

where $R$ is the set of the points in $X_{n+1}$ which are contained in neither contracting squares nor expanding squares of generation 1.

Proof. By Lemma 2.2 and Koebe's distortion theorem, it follows that there is a square $T \supset X_{n+1}$ with horizontal and vertical sides, such that $\operatorname{diam}(T) \preceq \operatorname{diam}\left(X_{n+1}\right)$. Moreover, $\operatorname{area}(T) \preceq \operatorname{area}\left(X_{n+1}\right)$. Let

$A_{\epsilon}^{\prime}=\{z \in \mathbb{C} \mid(2 k+1 / 2) \pi+4 \epsilon<\Im(z)<(2 k+3 / 2) \pi-4 \epsilon$ for some $k \in \mathbb{Z}\}$ and

$$
B_{\epsilon}^{\prime}=\{z \in \mathbb{C} \mid(2 k-1 / 2)+4 \epsilon<\Im(z)<(2 k+1 / 2) \pi-4 \epsilon \text { for some } k \in \mathbb{Z}\} .
$$


Let $R_{1}=T \backslash\left(A_{\epsilon}^{\prime} \cup B_{\epsilon}^{\prime}\right)$. It follows that

$$
\operatorname{area}\left(R_{1}\right) \preceq \epsilon \operatorname{area}(T) \preceq \epsilon \operatorname{area}\left(X_{n+1}\right) .
$$

Note that $\operatorname{diam}\left(X_{n+1}\right)>\delta$. By Lemma 2.2 and Koebe's distortion theorem, there is some uniform $C_{0}>0$ such that for any point $z \in E_{n+1}\left(S_{r^{\prime}}(1)\right)$, $S_{4 \epsilon}(z) \subset X_{n+1}$, where $r^{\prime}=r\left(1-C_{0} \epsilon / \delta\right)$. Let $R_{2}=X_{n+1} \backslash E_{n+1}\left(S_{r^{\prime}}(1)\right)$. By Lemma 2.2 and Koebe's distortion theorem again, we have

$$
\operatorname{area}\left(R_{2}\right) \preceq \epsilon \operatorname{area}\left(X_{n+1}\right) \text {. }
$$

Now it is sufficient to prove that $R \subset R_{1} \cup R_{2}$. In fact, for any point $z \in R$, there is some $\epsilon$-square $S=S_{\epsilon / 2}(c)$ which contains $z$. There are two possibilities. The first one is that $z \notin R_{1}$. Then $z \in X_{n+1} \cap\left(A_{\epsilon}^{\prime} \cup B_{\epsilon}^{\prime}\right)$. By the definition of $A_{\epsilon}^{\prime}$ and $B_{\epsilon}^{\prime}$, it follows that $S_{\epsilon}(c) \subset\left(A_{\epsilon} \cup B_{\epsilon}\right)$. Since $z$ belongs to neither expanding squares nor contracting squares, $S_{\epsilon}(c) \cap \partial X_{n+1} \neq \emptyset$. It follows that $S_{4 \epsilon}(z) \cap \partial X_{n+1} \neq \emptyset$ also. In particular, $z \in R_{2}$. This completes the proof of the lemma.

For an expanding square $S$ of generation $k$, let $\operatorname{Im}(S)=\left(E_{n+k+1} \circ\right.$ $\left.\Phi_{S}\right)(S)$.

Lemma 2.7. Let $r>0$ be small. Then for any $k \geq 1$ and any expanding square $S$ of generation $k$,

$$
\operatorname{area}(\operatorname{Im}(S) \backslash X) \preceq \epsilon \operatorname{area}(\operatorname{Im}(S))
$$

where $X$ is the union of all the expanding and contracting squares of generation $k+1$ generated by $S$, and

$$
\operatorname{area}(Y) \asymp \operatorname{area}(\operatorname{Im}(S)) \text {. }
$$

where $Y$ is the union of all the contracting squares of generation $k+1$ generated by $S$.

Proof. Let $S=S_{\epsilon / 2}(c)$. Let $\epsilon^{\prime}=\epsilon-\epsilon^{2}$ and $S^{\prime}=S_{\epsilon^{\prime} / 2}(c)$. Let $\operatorname{Im}\left(S^{\prime}\right)=$ $\left(E_{n+k+1} \circ \Phi_{S}\right)\left(S^{\prime}\right)$.

Consider the straight vertical lines and horizontal lines $x=2 k \pi$ and $y=2 k \pi$ for $k \in \mathbb{Z}$. These lines divide the complex plane into disjoint squares with side length $2 \pi$. Let $Z$ denote the collection of all those squares which are completely contained in $\operatorname{Im}(S)$. By Lemma 2.4 and induction, as 
$r>0$ is small, $\operatorname{Im}(S)$ lies in the far right of the complex plane, and by (12), $\left|D\left(E_{n+k+1} \circ \Phi_{S}\right)\right|$ is large in $S$. It follows that

$$
\bigcup_{T \in Z} T \supset \operatorname{Im}\left(S^{\prime}\right)
$$

for all $r>0$ small enough. Since $E_{n+k+1} \circ \Phi_{S}$ is defined in the $\epsilon$-neighborhood of $S$, by Koebe's distortion theorem, we have

$$
\operatorname{area}\left(\operatorname{Im}(S) \backslash \operatorname{Im}\left(S^{\prime}\right)\right) / \operatorname{area}(\operatorname{Im}(S)) \asymp \operatorname{area}\left(S \backslash S^{\prime}\right) / \operatorname{area}(S) \asymp \epsilon,
$$

and therefore

$$
\operatorname{area}\left(\operatorname{Im}(S) \backslash \bigcup_{T \in Z} T\right) \preceq \epsilon \operatorname{area}(\operatorname{Im}(S)) .
$$

Now consider any square $T \in Z$. Let $P$ and $Q$ be the union of all the contracting squares and expanding squares generated by $S$, and contained in $T$, respectively. It is clear that

$$
\operatorname{area}(P) \asymp \operatorname{area}(Q) \asymp \operatorname{area}(T)
$$

respectively, and

$$
\operatorname{area}(T \backslash(P \cup Q)) \preceq \epsilon \operatorname{area}(T) .
$$

This implies (13) and (14).

Now it is the time to prove

Lemma 2.8. There is a uniform $C>0$ such that for any $\epsilon>0$ small,

$$
\liminf _{r \rightarrow 0_{+}} \frac{\operatorname{area}\left(\Lambda_{r}(1)\right)}{\operatorname{area}\left(S_{r}(1)\right)}>1-C \epsilon .
$$

Proof. Let $T_{0}=S_{r}(1)$. Let $R$ be the set in Lemma 2.6 and $Y$ be the set in Lemma 2.7. Let $\Phi_{X_{n+1}}$ denote the inverse branch of $E_{n+1}$ which maps $X_{n+1}$ to $X_{0}=S_{r}(1)$. Let $R_{0}=\Phi_{X_{n+1}}(R)$, and $C_{0}=\Phi_{X_{n+1}}(Y)$. Let $T_{1}=T_{0} \backslash\left(R_{0} \cup C_{0}\right)$.

Let $k \geq 1$. Define $C_{k} \subset S_{r}(1)$ to be the set consisting of all those parameter values $\lambda$ such that there is a sequence of expanding squares $S_{1}, \ldots, S_{k}$ and a contracting square $S_{k+1}$ such that $S_{i}$ is generated by $S_{i-1}$ for $2 \leq i \leq k+1$, and $E_{n+i}(\lambda) \in S_{i}$ for $1 \leq i \leq k+1$.

Let $k \geq 2$. Define $T_{k} \subset S_{r}(1)$ to be the set consisting of all those parameter values $\lambda$ such that there is a sequence of expanding squares $S_{1}, \ldots, S_{k}$ 
such that $S_{i}$ is generated by $S_{i-1}$ for $2 \leq i \leq k$, and $E_{n+i}(\lambda) \in S_{i}$ for $1 \leq i \leq k$.

It is clear that $\left(T_{k+1} \cup C_{k}\right) \subset T_{k}$ for all $k \geq 0$. Define

$$
R_{k}=T_{k} \backslash\left(T_{k+1} \cup C_{k}\right) .
$$

We claim that

$$
\operatorname{area}\left(C_{k}\right) \succeq \operatorname{area}\left(T_{k}\right)
$$

holds for all $k \geq 1$, and

$$
\operatorname{area}\left(R_{k}\right) \preceq \epsilon \operatorname{area}\left(T_{k}\right)
$$

holds for all $k \geq 0$. Let us first see how the claim implies the lemma. In fact, from (15), it follows that

$$
S_{r}(1)=\left(\bigcup_{k \geq 0} R_{k}\right) \cup\left(\bigcap_{k \geq 0} T_{k}\right) \cup \Lambda_{r}(1) .
$$

From (15), (16), and (17), it follows that

$$
\operatorname{area}\left(\bigcap_{k \geq 0} T_{k}\right)=0
$$

and

$$
\operatorname{area}\left(\bigcup_{k \geq 0} R_{k}\right) \preceq \epsilon \operatorname{area}\left(S_{r}(1)\right) .
$$

The lemma then follows.

Now let us explain why the claim is true. Note that (16) comes directly from (14), Koebe's distortion theorem, and Remark 2.1. Let us see why (17) is true also. In fact, for $k=0$, it follows directly from Lemma 2.2 and Lemma 2.6. For $k \geq 1$, it follows from Lemma 2.7, Koebe's distortion theorem, and Remark 2.1.

\section{$\S 3 . \quad$ The parameters in $\Lambda_{r}(1)$ are hyperbolic}

LEMma 3.1. For any $\epsilon>0$ small, there is an $r_{0}>0$, such that for all $0<r<r_{0}$ and $\lambda \in \Lambda_{r}(1)$, the forward orbit $\left\{f_{\lambda}^{n}(0)\right\}_{n \geq 0}$ converges to some attracting periodic orbit. 
Proof. Let $\lambda \in \Lambda_{r}(1)$. By the construction of $\Lambda_{r}(1)$, there is a sequence of expanding squares $S_{1}, \ldots, S_{m-1}$, and a contracting square $S_{m}$, such that $S_{i+1}$ is generated by $S_{i}$ for $1 \leq i \leq m-1$, and $E_{n+i}(\lambda) \in S_{i}$ for $1 \leq i \leq m$. Let $p=n+m+2$. We will prove that $f_{\lambda}^{n}(0)$ is attracted to some attracting periodic orbit of period $p$. Let $z_{j}=f_{\lambda}^{j}(0)=x_{j}+i y_{j}$ where $x_{j}$ and $y_{j}$ are real numbers.

It is sufficient to prove that there is an open topological disk, say $U$, such that $\overline{f_{\lambda}^{p}(U)} \subset U$. It then follows that there is a unique attracting periodic orbit of period $p$ which attracts the forward orbit of every point in $U$. Now let us prove the existence of such $U$.

To show this, we claim that for any $R$ large, as long as $r>0$ is small, the disk $B_{R}\left(z_{p-1}\right)$ can be univalently pulled back to a small neighborhood of the origin by the map $f_{\lambda}^{p-1}(z)$ along the orbit $f_{\lambda}^{j}(0), 0 \leq j \leq p-1$.

Let us first show the lemma by assuming the claim. In fact, we may consider the pull back of $B_{1}\left(z_{p-1}\right)$ to a small neighborhood $V$ of the origin along the orbit $f_{\lambda}^{j}(0), 0 \leq j \leq p-1$. From the claim(by taking $R \gg 1$ ) and Koebe's distortion theorem, it follows that $V$ is like a Euclidean disk, more precisely,

$$
V \approx B_{r^{\prime}}(0)
$$

where $r^{\prime}=1 /\left|D f_{\lambda}^{p-1}(0)\right|$. But on the other hand, $f_{\lambda}\left(B_{1}\left(z_{p-1}\right)\right) \subset B_{r^{\prime \prime}}(0)$ where

$$
r^{\prime \prime}=C e^{-\left|x_{p-1}\right|}
$$

where $C>0$ is some uniform constant.

Notice that

$$
r^{\prime \prime} \ll r
$$

In fact, by Lemma 2.4, as $r>0$ is small, $p$ is large, and $x_{p-2} \succ 2^{p}$, and $x_{j+1}>2 x_{j}$ for $0 \leq j \leq p-3$. It then follows that

$$
\sum_{0 \leq j \leq p-2} x_{j}<2 x_{p-2} .
$$

But on the other hand, there is some $\epsilon_{0}>0$ dependent only on $\epsilon$ such that

$$
\left|x_{p-1}\right|>e^{\epsilon_{0} x_{p-2}} \gg x_{p-2}^{2}
$$


From this, and the fact that $\lambda$ is closed to 1 , we have

$$
\left|D f_{\lambda}^{p-1}(0)\right| \prec|\lambda|^{p} e^{x_{1}+\cdots+x_{p-2}} \prec|\lambda|^{p} e^{2 x_{p-2}} \prec e^{x_{p-2}^{2}} \ll e^{\left|x_{p-1}\right|} .
$$

This implies (19). From (18) and (19), we have $\overline{B_{r^{\prime \prime}}(0)} \subset V$. The lemma now follows by taking $U=V$ since as $r$ is small,

$$
\overline{f_{\lambda}^{p}(V)}=\overline{f\left(B_{1}\left(z_{p-1}\right)\right)} \subset \overline{B_{r^{\prime \prime}}(0)} \subset V .
$$

Now it remains to prove the claim. For given $R>0$, when $r>0$ is small, $x_{p-2} \gg 1$, it follows that the disk $B_{R}\left(x_{p-1}\right)$ can be univalently pulled back into the disk $B_{1 / 32}\left(x_{p-2}\right)$ by the map $f_{\lambda}(z)$ (This is because $f_{\lambda}(z)$ is univalent in the square $S_{1}\left(z_{p-2}\right)$, and $D f_{\lambda}\left(z_{p-2}\right)$ is large when $r>0$ is small). By Lemma 2.4, when $r>0$ is small, $\left|z_{j}\right|>8$ for all $2 \leq j \leq p-2$. Note that for $\xi, z \in B_{1 / 32}\left(z_{p-2}\right), \eta=|\xi-z| /|z|<1 / 32$ is small, we thus have

$$
|\log (\xi / \lambda)-\log (z / \lambda)|=\left|\log \left(1+\frac{\xi-z}{z}\right)\right|<\frac{\eta}{1-\eta}<1 / 32 .
$$

It follows from $(20)$ that $B_{1 / 32}\left(z_{p-2}\right)$ can be univalently pulled back into the disk $B_{1 / 32}\left(z_{p-3}\right)$. By induction, it follows that $B_{1 / 32}\left(x_{p-2}\right)$ can be univalently pull backed into the disk $B_{1 / 32}\left(x_{2}\right)$ by $f_{\lambda}^{p-4}$. But for $r>0$ small, that is, $\lambda$ close to 1 , the disk $B_{1 / 32}\left(x_{2}\right)$ can be univalently pull backed to some small neighborhood of the origin by $f_{\lambda}^{2}$. This proves the claim and thus completes the proof of Lemma 3.1.

The Main Theorem then follows from Lemmas 2.8 and 3.1.

Acknowledgement. The authors would like to thank the referee of a previous version of this paper. His or her many comments were very helpful in improving it.

\section{REFERENCES}

[1] I. N. Baker and P. J. Rippon, Iteration of exponential functions, Ann. Acad. Sci. Fenn., 9 (1984), 49-77.

[2] R. Devaney, Julia sets and bifurcation diagrams for exponential maps, Bull. Amer. Math. Soc., 11 (1984), 167-171.

[3] R. Devaney, Structure Stability of $\exp (z)$, Proc. Amer. Math. Soc., 94 (1985), no. 3, 545-548. 
[4] R. Devaney, L. Goldberg, and J. Hubbard, A dynamical approximation to the exponential map by polynomials, Prepint M.S.R.I. (1985).

[5] R. Devaney, N. Fagella and X. Jarque, Hyperbolic components of the complex exponential family, Fund. Math., 174 (2002), no. 3, 193-215.

[6] R. Devaney and M. Krych, Dynamics of $\exp (z)$, Ergodic Theory \& Dynamical System (1984), 35-52.

[7] A. Douady and L. Goldberg, The nonconjugacy of certain exponential functions, M.S.R.I. publ. 10, Springer-Verlag, New York, 1988.

[8] E. Ghys, L. Goldberg, and D. Sullivan, On the Measurable Dynamics of $z \rightarrow e^{z}$, Ergodic Theory \& Dynamical System, 5 (1985), 329-335.

[9] M. Lyubich, Measurable Dynamics of the Exponential, Siberian J. Math., 28 (1987), $111-127$.

[10] M. Misiurewicz, On iterates of $e^{z}$, Ergodic Theory \& Dynamical System, 1 (1981), $103-106$.

[11] C. McMullen, Area and Hausdorff dimension of Julia sets of entire functions, Trans. Amer. Math. Soc., 300 (1987), no. 1, 329-342.

[12] W. Qiu, Hausdorff dimension of the M-set of $\lambda \exp (z)$, Acta Math. Sinica (N.S.), 10 (1994), no. 4, 362-368.

[13] M. Rees, The Exponential Map is not Recurrent, Math. Z., 191 (1986), 593-598.

[14] M. Rees, Positive Measure Sets of Ergodic Rational Maps, Ann. Scient. éc. Norm. Sup., 4 série, t. 19, 1986, pp. 383-407.

[15] D. Schleicher, Attracting Dynamics of Exponential Maps, Ann. Acad. Sci. Fenn. Math., 28 (2003), 3-34.

[16] Z. Ye, Structural instability of exponential functions, Trans. Amer. Math. Soc., 344 (1994), no. 1, 379-389.

[17] J. Zhou and Z. Li, Structural instability of mapping $z \rightarrow \lambda \exp (z)\left(\lambda>e^{-1}\right)$, Sci. China. Ser. A, 301 (1989), 1153-1161.

\author{
Xiumei Wang \\ Department of Computer Science and Information Technology \\ JiangSu Teachers University of Technology \\ Changzhou, 213001 \\ P. R. China \\ wxmei@jstu.edu.cn \\ Gaofei Zhang \\ Department of Mathematics \\ Nanjing University \\ Nanjing, 210093 \\ P. R. China \\ zhanggf@hotmail.com
}

